\title{
Shaping planetary nebulae with jets in inclined triple stellar systems
}

\author{
Muhammad Akashi and Noam Soker \\ Department of Physics, Technion - Israel Institute of Technology, Haifa 32000, Israel; \\ akashi@ph.technion.ac.il, soker@physics.technion.ac.il
}

\begin{abstract}
We conduct three-dimensional hydrodynamical simulations of two opposite jets launched obliquely to the orbital plane around an asymptotic giant branch (AGB) star and within its dense wind, and demonstrate the formation of a 'messy' planetary nebula (PN), namely, a PN lacking any type of symmetry (highly irregular). In building the initial conditions we assume that a tight binary system orbits the AGB star, and that the orbital plane of the tight binary system is inclined to the orbital plane of binary system and the AGB star. We further assume that the accreted mass onto the tight binary system forms an accretion disk around one of the stars, and that the plane of the disk is in between the two orbital planes. The highly asymmetrical lobes that we obtain support the notion that messy PNe might be shaped by triple stellar systems.
\end{abstract}

Keywords. binaries: close - planetary nebulae - jets

\section{Introduction}

The notion that binary stellar systems or planetary systems shape planetary nebulae (PNe) has gained huge support over the years, both from theoretical considerations that point to the difficulties of models based on single asymptotic giant branch (AGB) stellar progenitors to shape $\mathrm{PNe}$, and from a large number of observations and their analysis. In most cases of shaping by binary interaction, jets are involved. A relatively small number of papers raise the possibility that triple stellar systems are behind the morphologies of some PNe. In general there are two orbital planes. One is that of the more tight binary system, and the second one is that of the tight binary system motion around the center of mass with the third star. If the two orbital planes coincides, the interaction leads to a mass loss geometry that has a plane of symmetry, even if the mass loss geometry departs from axi-symmetry. Binary systems, e.g., if the interaction time scale is shorter than the orbital period or if the orbit is eccentric, can also cause departure from axisymmetry.

If the two orbital planes of the triple system are inclined to each other, the morphology of the descendant PN is likely to possess no symmetry plane. The descendant PN is actually likely to lack any symmetry; nor point-symmetry, nor axial-symmetry, and nor mirror symmetry. We term this a 'messy PN'.

Specifically, we consider the flow structure described by Soker (2004), where a tight binary system orbits an AGB star and accretes mass from the AGB wind. Because the orbital plane of the tight binary system is not parallel to the orbital plane around the AGB star, the jets' axis is not perpendicular to the orbital plane. We describe the initial setting and the numerical code in section 2. We then describe the numerical results in section 3. We summarize our results and call for the search for triple stellar systems in the center of messy PNe. 


\section{Numerical Set-up}

\subsection{Initial conditions}

The two orbital planes, that of the tight binary system and that of the triple stellar system are inclined to each other. The tight binary system accretes mass from the wind. Due to the orbital motion of the two stars of the tight binary system around their mutual center of mass, the accreted mass possesses specific angular momentum that leads to the formation of an accretion disk around one (or two) of the stars. The plane of the accretion disk is close to being parallel to the orbital plane of the tight binary system. The accretion disk launches two opposite jets that are almost perpendicular to the orbital plane of the tight binary system.

We simulate two cases which differ in the inclination angle $\phi$ of the jets' axis to the line perpendicular to the orbital plane of the triple system. In the first case $\phi=20^{\circ}$ and in the second case $\phi=30^{\circ}$. The other parameters are identical for the two runs and are as follows. The orbital separation of the jets' origin (the location of the right binary system) to the center of the AGB star is $a_{123}=30 \mathrm{AU}$. The orbital period is $P_{123}=67 \mathrm{yrs}$ corresponding to a total mass of the triple stellar system of $M_{123}=M_{1}+M_{2}+M_{3}=5 M_{\odot}$. (see Fig. 1).

At the beginning of each simulation the slow AGB wind fills the grid. The mass-loss rate and velocity of the slow AGB wind are $\dot{M}_{w}=5 \times 10^{-5} M_{\odot} \mathrm{yr}^{-1}$ and $v_{w}=10 \mathrm{~km} \mathrm{~s}^{-1}$, respectively.

At $t=0$ we start to launch two jets, along the $+z$ and $-z$ directions. The jets are conical, with a half opening angle of $\alpha_{j}=30^{\circ}$. The jets opening angle influences the morphology of the nebula. However, we have limited numerical resources and cannot study more cases at this time. The initial velocity of the jets are $v_{j}=500 \mathrm{~km} \mathrm{~s}^{-1}$, that is about equal to the escape speed from a main sequence star. The mass-loss rate into the two jets together is $\dot{M}_{2 j}=2 \times 10^{-7} M_{\odot}=0.004 \dot{M}_{w}$. This corresponds to about $10 \%$ of the accretion rate from the wind.

\subsection{The numerical code}

Our simulations are performed by using version 4.0-beta of the FLASH code. We employ a full 3D adaptive mesh refinement (AMR) (10 levels; $\left.2^{13}\right)$ using a Cartesian grid $(x, y, z)$ with outflow boundary conditions at all boundary surfaces. The center of the grid is at $(0,0,0)$. The AGB star is at a permanent place on the grid, it is placed at $(0,0,-$ $\left.a_{123} \times \cos (\phi)\right)$.

The slow wind and the ejected jets start with a temperature of $1000 \mathrm{~K}$. The initial jets' temperature has no influence on the results (as long as it is highly supersonic) because the jets rapidly cool due to adiabatic expansion.

\section{Results}

We show three-dimensional density map for the case with inclination angle $\phi=30$ (see Fig. 2).

\section{Summary}

As possible examples for PNe that might have been shaped by a triple stellar system Soker (2004) listed several PNe, including IC 2149, NGC 6210, and NGC 1514, that we also suggest here were shaped by a triple stellar progenitor.

N.S. is supported by the Charles Wolfson Academic Chair. 


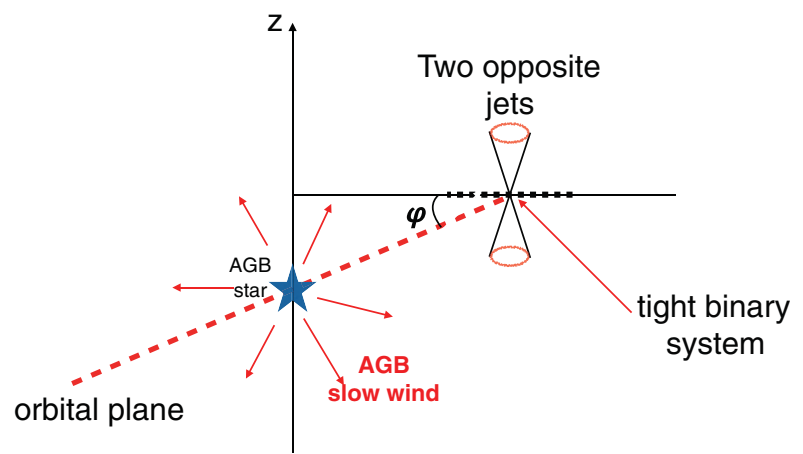

Figure 1. A schematic drawing of the outflow from the triple stellar system. The orbital plane of the tight binary system and the one of the triple system are inclined by an angle $\phi$. The tight binary system accretes mass from the AGB wind and launches jets as indicated.

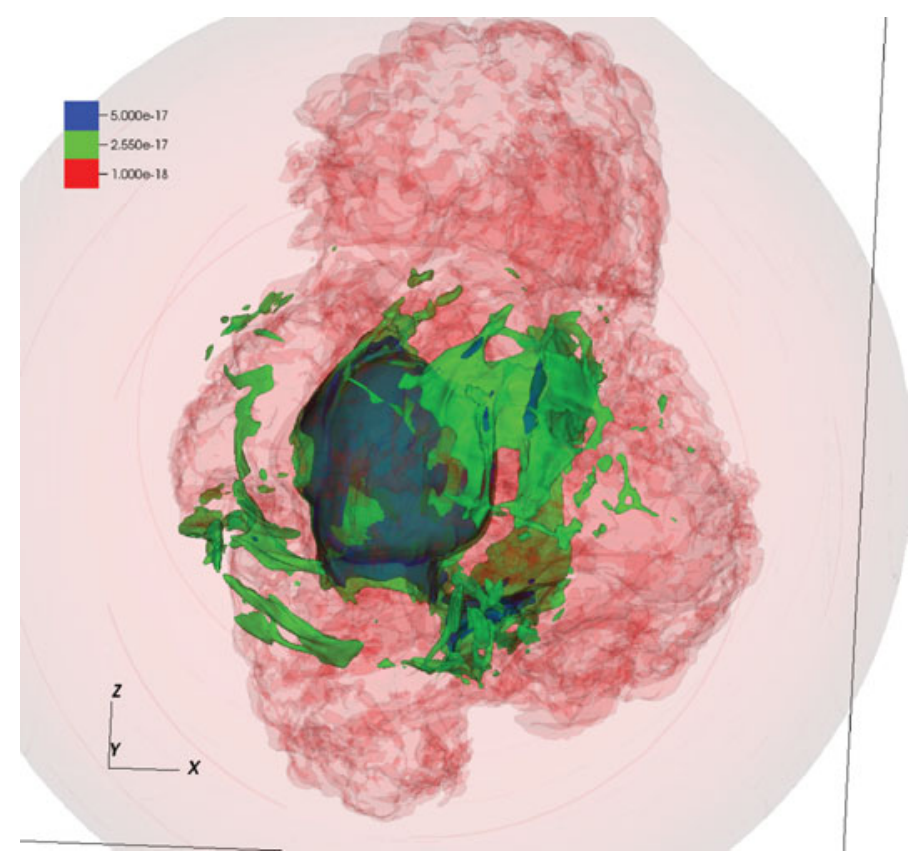

Figure 2. Three dimensional density map of the case $\phi=30$.

\section{References}

Soker, N. 2004, MNRAS, 350, 1366

Soker, N. 2016, MNRAS, 455, 1584

\section{Discussion}

De MARCO: What causes the misalignment of the plane of the accretion disk and the plane of the orbit (the AGN orbit)?

AKASHI: The misalignment of the tight orbital plane is just an initial condition in our simulation. The accreted mass gains angular momentum from the tight binary. See Soker (2004).

ToDT: Can you please comment on the plausibility of the assumed properties of the 
triple system, e.g. what are the eccentricities, how stable is such configuration over the stellar lifetime, what is the reason for the jet?

AKASHI: The properties and the parameters were chosen after an accurate calculation of the condition of accretion. The orbits are circular and stable. The reason for the jet is accretion from the AGB wind onto the tight binary, so you have disk and jet.

Schoenberner: What is the time scale of these simulations? For applications to real objects one has to include the radiation field of the central source: The thermal pressure of ionized gas will certainly smooth the distorted envelope. 